\title{
Genomic DNA methylation profiling indicates immune response following thermal ablation treatment for HBV-associated hepatocellular carcinoma
}

\author{
YANAN ZHAO ${ }^{1,2}, \mathrm{KANG} \mathrm{LI}^{1}$, JIANPING SUN ${ }^{1}$, NING HE ${ }^{2}$, PENG ZHAO $^{2}, \mathrm{CHAORAN} \mathrm{ZANG}^{1,2}$, \\ XIAOZHEN YANG ${ }^{2}$, CAIXIA HU ${ }^{2}$, JIANG LONG ${ }^{2}$, HONGHAI ZHANG ${ }^{2}$, \\ QI WANG ${ }^{1,2}$, YAN ZHAO ${ }^{3}$ and YONGHONG ZHANG ${ }^{1,2}$ \\ ${ }^{1}$ Research Center for Biomedical Resources; ${ }^{2}$ Interventional Therapy Center for Oncology; \\ ${ }^{3}$ Clinical Detection Center, Beijing You'an Hospital, Capital Medical University, Beijing 100069, P.R. China
}

Received October 15, 2019; Accepted April 15, 2020

DOI: 10.3892/ol.2020.11636

\begin{abstract}
Hepatitis B virus (HBV)-associated hepatocellular carcinoma (HCC) is the most common type of liver cancer in China. Thermal ablation is one of the main strategies for HCC treatment. However, few studies have investigated the properties of the immune response following thermal ablation thus far. In the present study, five subjects with HBV-associated HCC were recruited from The Beijing You'an Hospital. Peripheral blood mononuclear cells (PBMCs) were collected at three time points: Prior to thermal ablation (PR), 1-3 days post-ablation (P1) and 5-7 days post-ablation (P7). An Illumina $850 \mathrm{~K}$ methylation microarray was employed to determine the DNA methylation profile of each sample. Data were analyzed using different methylation probes with the Bioconductor package in R. Following annotation of different methylation CG sites (CGs), the associated genes were subjected to an Ingenuity Pathway Analysis. A total of 3,000 significantly different CGs (adjusted $\mathrm{P}<0.05$; $\log$ (fold-change) $>0.5$ ) were identified within the PR, P1 and P7 time points. Of these,
\end{abstract}

Correspondence to: Dr Yonghong Zhang, Interventional Therapy Center for Oncology, Beijing You'an Hospital, Capital Medical University, 8 XiTouTiao Youanmen Wai, Beijing 100069, P.R. China E-mail: 13810108505@163.com

Dr Yan Zhao, Clinical Detection Center, Beijing You'an Hospital, Capital Medical University, 8 XiTouTiao Youanmen Wai, Beijing 100069, P.R. China

E-mail: 18911380390@163.com

Abbreviations: HBV, hepatitis B virus; HCC, hepatocellular carcinoma; PBMCs, peripheral blood mononuclear cells; CGs, CG sites; RFA, radiofrequency ablation; MWA, microwave ablation; TACE, transarterial chemoembolization; IPA, Ingenuity Pathway Analysis; FC, fold-change; APC, antigen-presenting cell; Th1, $\mathrm{T}$ helper cell 1; Th2, T helper cell 2; p70S6K, p70 ribosomal S6 kinase; CXCR4, C-X-C motif chemokine receptor 4

Key words: HCC, PBMCs, DNA methylation, immune response
744 (24.8\%) sites increased between the PR and P1 time points but gradually decreased at the $\mathrm{P} 7$ time point. The remaining 2,256 (75.2\%) sites decreased between the PR and P1 time points gradually increased at the P7 time point. Following gene annotation of different CGs on the promoter, signaling pathways analysis demonstrated that 'p70S6K signaling', 'CXCR4 signaling', 'dendritic cell maturation', 'production of nitric oxide and reactive oxygen species in macrophages' pathways were activated at the P7 time point. The present study suggested that PBMC DNA methylation had changed soon after thermal ablation for subjects with HBV-associated HCC, and systemic immune responses were activated, particularly at the $\mathrm{P} 7$ time point.

\section{Introduction}

Hepatocellular carcinoma (HCC) is the most common form of liver cancer, the sixth most commonly diagnosed cancer, and the fourth leading cause of cancer-associated mortality worldwide (1). In China, HCC is the third leading cause of cancer-associated mortality (2). Moreover, the mortality rate of hepatitis B virus (HBV)-associated liver cancer was 16.42 per 100,000 people in 2016 (3). The annual disability-adjusted life-years of liver cancer caused by HBV in China have been consistently higher than the global level (3).

While surgical resection is the most common method for HCC treatment, radiofrequency ablation (RFA) and microwave ablation (MWA) have attracted increasing interest due to the reduced trauma and faster recovery observed with these approaches (4). Additionally, both RFA and MWA are accessible to subjects who are not eligible for surgical resection $(5,6)$. Notably, no difference has been observed in the overall survival rate and disease-free survival rate in subjects with early-stage HCC between ablation and surgical resection $(7,8)$. An alternative tumor-specific target treatment should generate a tumor-specific immune response in patients with cancer (9). Previous clinical studies suggested that RFA induced the expression of tumor-associated antigen as well as the activation of the immune response, which not only increased the number of tumor-specific immune cells, but also the frequency 
of immune cells specific for recall antigens (10-12). However, whether the systemic immune response can be activated after ablation remains unclear.

DNA methylation mediates primary epigenetic regulation of genome function. Previous studies indicated that DNA methylation was involved in the development of immune cells (13), T lymphocyte function, persistent inflammation, HCC progression (14-16) and the effector phase of chronic viral infection (17). The inhibition of de novo DNA methylation programs combined with immune checkpoint blockade can facilitate the control of chronic viral infection and tumor growth (18). Our previous study demonstrated that peripheral blood mononuclear cells (PBMCs) DNA methylated alterations were enriched in immune-associated canonical pathways and had immune modulation functions during HCC progression (19). Thus, it is essential to investigate the dynamic immune response profile following thermal ablation by DNA methylation, as well as the potential effective immune response mechanism.

To the best of the authors' knowledge, few studies have investigated the effects of the systemic immune response and immune-activated time points following thermal ablation for HCC. The present study established the PBMC DNA methylation profiles for HBV-associated HCC in order to describe the epigenetic changes associated with the immune response following thermal ablation treatment.

\section{Materials and methods}

Sample collection. In the present study, five subjects with HCC (The nomenclature of the subjects were represented by 'M + number', thereafter referred to as M1, M2, M3, M4 and M5) were recruited at Beijing You'an Hospital of the Capital Medical University from January 2018 and March 2018. The diagnosis of HCC was based on the European Association for the Study of the Liver Clinical Practice Guidelines (20), and the classification of HCC stages was based on the Barcelona Clinic Liver Cancer staging system (21). The following inclusion criteria were used: i) Liver biopsy or film degree exam diagnosed as HCC; ii) subjects positive for HBV surface antigen; iii) liver cirrhosis classified as Child-Pugh class A; iv) single HCC nodule $\leq 5 \mathrm{~cm}$; and v) absence of extrahepatic metastasis. The exclusion criteria were as follows: i) Subjects undergoing treatment, such as thermal ablation, surgical resection, chemotherapy, radiotherapy, gene therapy, adoptive cellular immunotherapy, or any other treatments for HCC; ii) subjects with coexistent hematological disorders, serious or active infection before treatment; iii) subjects with other types of cancer; iv) combinations with other virus infections and autoimmune diseases; v) no procedure-associated complications occurred; and vi) subjects undergoing other interventional procedures during hospitalization, such as biliary drainage or cyst puncture drainage. No subjects included in the present study had received HCC-associated treatment. All subjects had undergone abdominal CT or abdominal MRI before and after thermal ablation (thereafter referred to as ablation). The present study was approved by The Ethics Committee of Beijing You'an Hospital, Capital Medical University. Written informed consent was obtained from all subjects. The present study was performed according to the guidelines of the Helsinki Declaration.
A total of $10 \mathrm{ml}$ whole-blood samples were collected prior to thermal ablation (PR), 1-3 days post-ablation (P1), and 5-7 days post-ablation (P7) following the first ablation treatment (among them, M3 subject underwent two ablation treatment, and other subjects underwent one ablation treatment in the hospitalization). PBMCs were isolated by Ficoll density gradient within $6 \mathrm{~h}$ after peripheral blood collection, then stored in liquid nitrogen until use.

Interventional treatments. Before transarterial chemo-embolization (TACE; microguide wire and microcatheter; Asahi Intecc, Co., Ltd.), a percutaneous liver biopsy was taken. RFA (RF electrode needle; AngioDynamics, Inc.) or MWA (microwave ablation needle; Nanjing Eco Microwave System, Co., Ltd.) was performed under local anesthesia 1 week after TACE. The power and duration of RFA or MWA are presented in Table I. The aforementioned treatments were performed by an interventional radiologist with $>5$ years of experience. Abdominal CT was used to evaluate the ablation effect by all radiologists in The Interventional Therapy Center for Oncology.

Illumina BeadChip $850 \mathrm{~K}$ analysis and methylation assay. DNA was extracted from PBMCs using a QIAamp DNA Mini kit (Qiagen $\mathrm{GmbH}$ ) according to the manufacturer's protocol. DNA purity was assessed by measuring the $\mathrm{A}_{260} / \mathrm{A}_{280}$ ratio using a NanoDrop ${ }^{\mathrm{TM}}$ instrument (Thermo Fisher Scientific, Inc.). DNA integrity was evaluated via agarose gel electrophoresis. An intense band within the high-molecular weight range ( $>10 \mathrm{~kb}$ ) was required to pass the quality control assessment. Bisulfite conversion was performed according to the manufacturer's recommendations in the EZ DNA Methylation kit (Zymo Research Corp.), followed by Illumina $850 \mathrm{~K}$ BeadChip analysis (Infinium Methylation EPIC BeadChip v.1.0 (8 samples/chip); Illumina, Inc.). DNA methylation levels was detected using Illumina Infinium HD Methylation Assay (Infinium Methylation EPIC BeadChip kit v.1.0 (16 samples); Illumina, Inc.) according to the manufacturer's protocol.

Statistical analysis. Illumina arrays were analyzed using Bioconductor package in R (22-25). Raw data were kept for probes with a mean detection value of $\mathrm{P}<0.05$. Probes on the $\mathrm{X}$ or $\mathrm{Y}$ chromosome were filtered out in order to mitigate sex effects, as well as probes with single nucleotide polymorphisms and probes that aligned to multiple locations $(24,25)$. Intra-array normalization adjusting biased data was performed using preprocess Quantile (24). The present study corrected for batch effects after normalization and filtering using the sva Bioconductor package in $\mathrm{R}$ (26). The $\beta$-values of the normalized data were used for downstream statistical analysis. Differentially methylated CG sites (CGs) at PR, P1 and P7 time points were identified using one-way ANOVA with the lmFit function in the Bioconductor package Limma (23) and correcting for multiple testing using Benjamini-Hochberg (adjusted $\mathrm{P}<0.05$ ), and $\log (\mathrm{FC}) \mathrm{l}>0.5$ was also used to indicate the significant difference, where FC is the fold-change (23). Pearson correlation analyses were performed to identify correlations among the three-time points $(r>0.8$ or $r<-0.8)$. Heatmaps and boxplots were generated using the pheatmap (https://CRAN.R-project.org/package=pheatmap) and ggplot2 
Table I. Demographic and clinical data of five patients with hepatitis B virus-associated hepatocellular carcinoma undergoing thermal ablation.

\begin{tabular}{|c|c|c|c|c|c|}
\hline Subjects & $\mathrm{M}^{\mathrm{a}} 1$ & $\mathrm{M}^{\mathrm{a} 2}$ & $\mathrm{M}^{\mathrm{a} 3}$ & $\mathrm{M}^{\mathrm{a}} 4$ & $\mathrm{M}^{\mathrm{a} 5}$ \\
\hline Time point-PR ${ }^{\mathrm{b}}$ & $2018 / 1 / 8$ & $2018 / 1 / 24$ & $2018 / 2 / 23$ & 2018/3/16 & $2018 / 2 / 23$ \\
\hline Time point-P $1^{\mathrm{c}}$ & 2018/1/18 & $2018 / 1 / 30$ & 2018/3/1 & $2018 / 3 / 21$ & $2018 / 3 / 2$ \\
\hline Time point- $\mathrm{P} 7^{\mathrm{d}}$ & $2018 / 1 / 22$ & $2018 / 2 / 5$ & $2018 / 3 / 7$ & $2018 / 3 / 26$ & $2018 / 3 / 6$ \\
\hline Age & 48 & 62 & 46 & 58 & 50 \\
\hline Sex & Male & Female & Male & Male & Male \\
\hline Alcohol & Abstaining for 6 years & No & $\begin{array}{c}\text { Consuming for the past } \\
5 \text { years }\end{array}$ & No & No \\
\hline Smoking, years & No & 30 & 26 & No & No \\
\hline Cirrhotic morphology & Yes & Yes & Yes & Yes & Yes \\
\hline Prior treatment & No & No & No & No & No \\
\hline Tumor nodules, $\mathrm{n}$ & 1 & 1 & 4 & 1 & 1 \\
\hline Maximum diameter, cm & 4.4 & 2.5 & $4.5,1,1,2$ & 2.2 & 2.7 \\
\hline $\begin{array}{l}\text { Ablation region maximum } \\
\text { diameter, } \mathrm{cm}\end{array}$ & 5.45 & 7.15 & $6.08,2.14$ & 8.92 & 6.23 \\
\hline Tumor size treated, $\mathrm{cm}$ & 4.4 & 2.5 & $4.5,1$ & 2.2 & 2.7 \\
\hline Complete ablation or not ${ }^{\mathrm{e}}$ & Yes & Yes & No & No & Yes \\
\hline Total ablation number ${ }^{\mathrm{f}}$ & 1 & 1 & 2 & 1 & 1 \\
\hline Vascular invasion & No & Yes & No & Yes & No \\
\hline Distant metastasis & No & No & No & No & No \\
\hline BCLC $^{\mathrm{g}}$ & A & $\mathrm{C}$ & $\mathrm{B}$ & $\mathrm{C}$ & A \\
\hline Surgery duration, min & 85 & 80 & 100 & 160 & 120 \\
\hline Relapse & No & No & No & Yes & No \\
\hline
\end{tabular}

${ }^{\mathrm{a}}$ Nomenclature of the subjects; ${ }^{\mathrm{b} P r i o r}$ to thermal ablation; ${ }^{\mathrm{c}} 1-3$ days post-ablation; ${ }^{\mathrm{d} 5-7}$ days post-ablation; ${ }^{\mathrm{e}}$ Complete ablation or incomplete ablation at the sample collection; ${ }^{\mathrm{f}}$ The total number of ablation in this hospitalization; ${ }^{\circ}$ Classification of hepatocellular carcinoma stages based on the BCLC staging system. PR, prior to thermal therapy; P1, 1-3 days post-ablation; P7, 5-7 days post-ablation; BCLC, Barcelona Clinic Liver Cancer.

(https://CRAN.R-project.org/package=ggplot2) package in R. After the annotation of differentially methylated CGs on the promoter, the mean $\beta$-value of those CGs was calculated to predict positive or negative gene expression. Pathway analysis of genes was determined by Gene Ontology analysis using Ingenuity Pathway Analysis (IPA; Qiagen GmbH), Fisher's exact test was used to calculate P-values, determining the probability between genes and the canonical pathways, and any pathway with $-\log (\mathrm{P})>1$ and at least three genes were included in the analysis. The age of those patients is expressed as the mean \pm standard deviation.

\section{Results}

Clinical characteristics. The demographic and clinical characteristics of the five subjects are presented in Table I. The mean age was $52.80 \pm 6.87$ years. Parameters which could impact the distribution of DNA methylation, such as age, sex, drinking and smoking, were eliminated by paired analysis with the samples of each subject before and after ablation treatment.

Distribution of DNA methylation differs at the PR, $P 1$ and $P 7$ time points. The present study delineated differentially methylated CGs among the PR, P1 and P7 time points using the Bioconductor package Limma (23). A total of 119,440 differentially methylated CGs were identified (adjusted $\mathrm{P}<0.05$ ): 115,166 different CGs between the PR and P1 time points, 84,330 CGs between the PR and P7 time points, and 84,673 CGs between the P1 and P7 time points. T-distributed stochastic neighbor embedding of those 119,440 CGs exhibited a good separation effect among the three time points (Fig. 1A).

Paired analysis with Benjamini-Hochberg correction (adjusted $\mathrm{P}<0.05 ;|\log (\mathrm{FC})|>0.5$ ) was performed. Finally, there were 2,779 differentially methylated sites between the PR and P1 time points (Fig. 1B), 374 differentially methylated sites between the P1 and P7 time points (Fig. 1C), and 204 differentially methylated sites between the PR and P7 time points (Fig. 1D). The clustering analysis demonstrated that all those different CGs could well distinguish those time points.

Immune activation at the PI time point is associated with systemic stress responses. Next, 3,000 differently methylated CGs (adjusted $\mathrm{P}<0.05 ;|\log (\mathrm{FC})|>0.5)$ in all three groups were focused on. The mean methylation levels of those 3,000 CGs were decreased between the PR and P1 time points, but increased at the P7 time point (Fig. 2A). Among the 3,000 CGs, there were $744(24.8 \%$; 744/3,000) sites with increased methylation between the PR and P1 time points, which gradually 
A

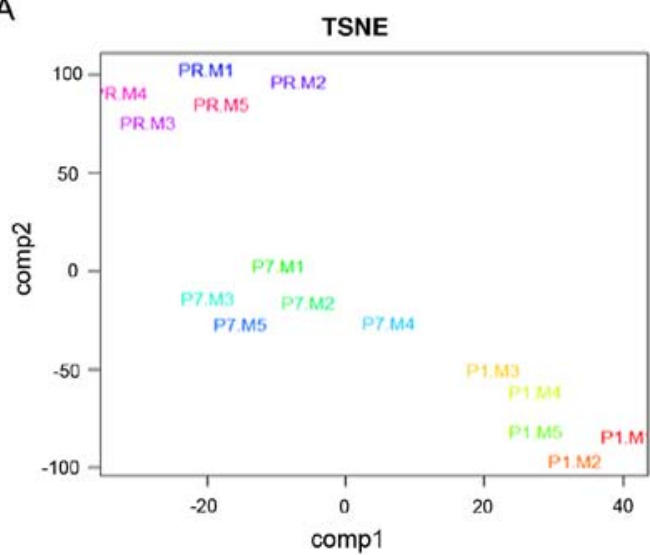

C

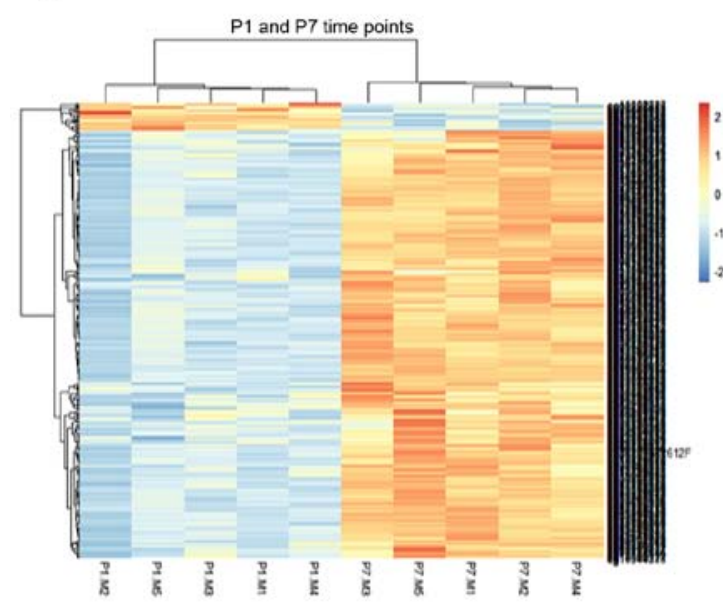

B

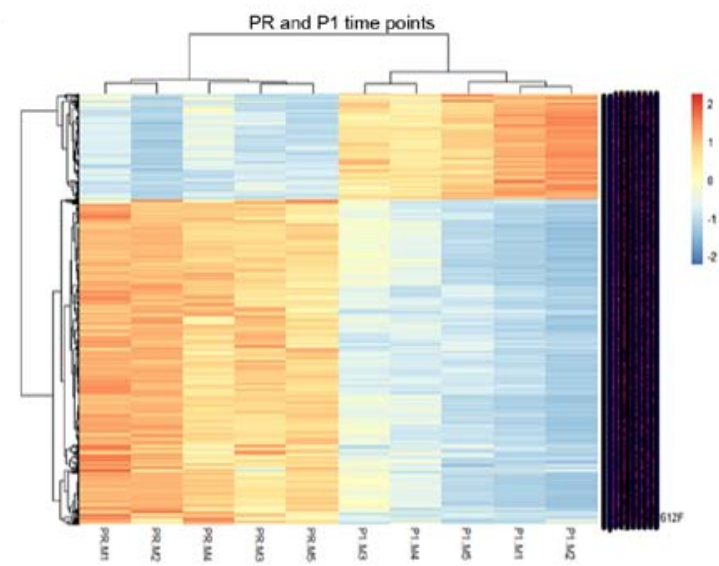

D

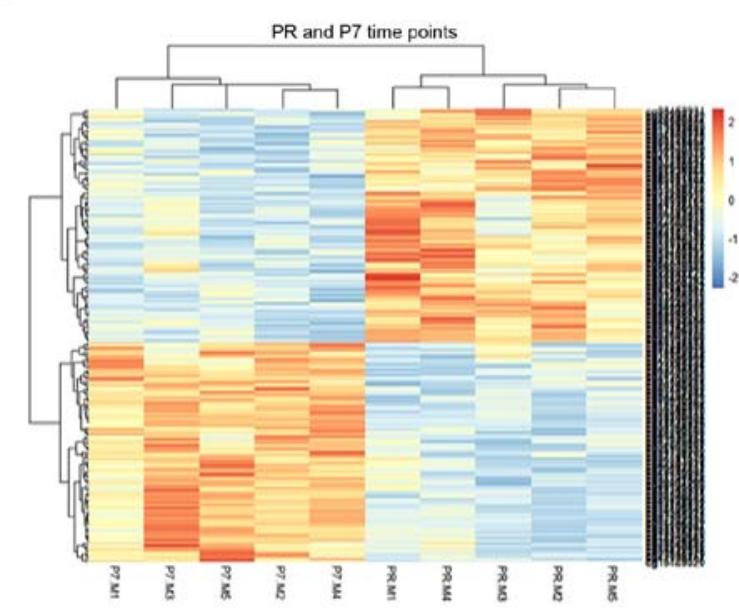

Figure 1. Differentially methylated CGs at the PR, P1 and P7 time points. (A) One-way ANOVA was used to compare differentially methylated CGs among the three time points. Distributional characteristics of the 119,440 different CGs are displayed by TSNE. (B) Heatmap of hierarchical clustering by 2,779 differentially methylated CGs between the PR and P1 time points, the color indicates the CGs methylation level, the deep color represents greater expression (red denotes hypermethylation; blue denotes hypomethylation). (C) Heatmap of hierarchical clustering by 374 differentially methylated CGs between the P1 and P7 time points, the color indicates the CGs methylation level, the deep color represents greater expression (red denotes hypermethylation; blue denotes hypomethylation). (D) Heatmap of hierarchical clustering by 204 differentially methylated CGs between the PR and P7 time points, the color indicates the CGs methylation level, the deep color represents greater expression (red denotes hypermethylation; blue denotes hypomethylation). Adjusted $\mathrm{P}<0.05$; $\mid \log ($ fold-change) $\mid>0.5$. CGs, CG sites; TSNE, T-distributed stochastic neighbor embedding; PR, prior to thermal therapy; P1, 1-3 days post-ablation; P7, 5-7 days post-ablation.

decreased at the P7 time point (Fig. 2B). At the same time, there were $2,256(75.2 \% ; 2,256 / 3,000)$ sites with decreased methylation between the PR and P1 time points, which gradually increased at the P7 time point (Fig. 2C).

Gene annotation of differentially methylated sites located on the promoter indicated that there were 759 different genes between the PR and P1 time points, and 167 different genes between the P1 and P7 time points. The signaling pathways associated with these genes were then identified using IPA. The results were filtered in order to select immune-associated pathways, which identified 46 signaling pathways between the PR and P1 time points, 27 signaling pathways between the P1 and $\mathrm{P} 7$ time points $(-\log (\mathrm{P})>1$, with at least three genes in one pathway).

For the PR and P1 time points, several systemic stress response pathways were identified, such as 'neuroinflammation signaling pathway' and 'antioxidant action of vitamin C'. Innate and adaptive immune pathways were also observed, such as 'T cell exhaustion signaling pathway', 'production of nitric oxide and reactive oxygen species in macrophages' and 'activation of IRF by cytosolic pattern recognition receptors'. Moreover, the 'FAT10 cancer signaling pathway' was activated. However, T lymphocyte co-stimulation ('CD28 signaling in T helper cells' and 'CD40 signaling'), activation ('PKC $\theta$ signaling in T lymphocytes'), migration ('CXCR4 signaling') and the 'Th1 pathway' were suppressed (Fig. 2D).

At the P1 and P7 time points, several systemic stress response pathways were suppressed, such as the 'adrenomedullin signaling pathway' and the 'NRF2-mediated oxidative stress response'. Several innate and adaptive immune pathways that were activated at the P1 time point were suppressed at the P7 time point, such as ' $\mathrm{T}$ cell exhaustion signaling pathway' and 'production of nitric oxide and reactive oxygen species in macrophages'. The change trends of 19 other pathways, including 'dendritic cell maturation' and 'natural killer cell signaling', were unclear (Fig. 2E).

Activation of the adaptive immune response at the P7 time point. Following the gene annotation and signaling pathway analysis, there were 184 different genes, and 29 signaling 
A

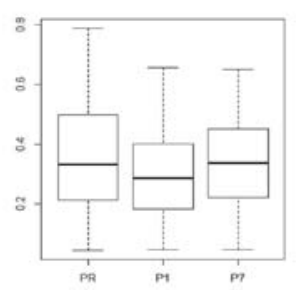

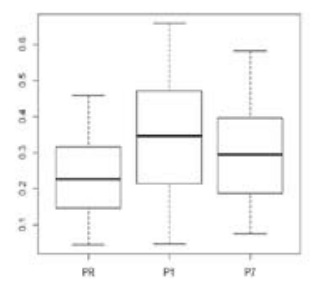

c

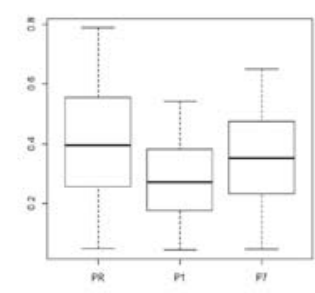

D

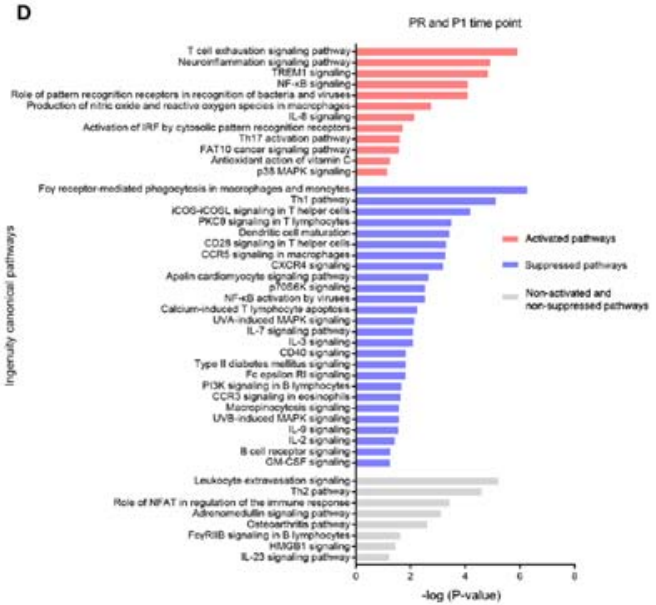

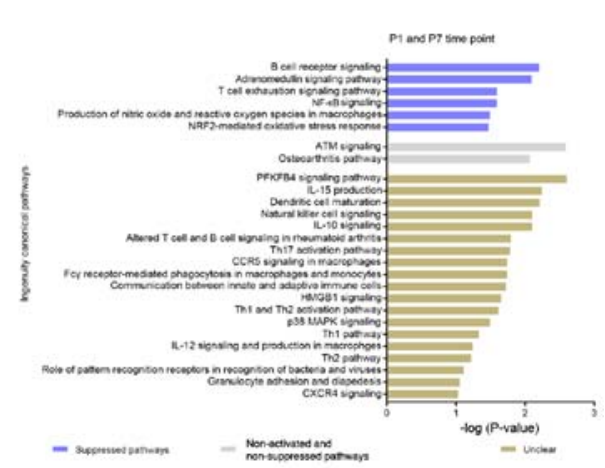

Figure 2. Change trend of mean methylation level and pathway analysis at the PR, P1, P7 time points. (A) Boxplot of DNA methylation mean $\beta$-values of 3,000 CGs (adjusted P<0.05; $|\log (\mathrm{FC})|>0.5$ ) among the PR, P1 and P7 time points. (B) Boxplot of DNA methylation mean $\beta$-values of 744 (24.8\%; 744/3,000) increased CGs (adjusted P<0.05; $\log (\mathrm{FC}) \mid>0.5$ ) between the PR and P1 time points, which were decreased at the P7 time point. (C) Boxplot of DNA methylation mean $\beta$-values of $2,256(75.2 \% ; 2,256 / 3,000)$ decreased CGs (adjusted $\mathrm{P}<0.05 ;|\log (\mathrm{FC})|>0.5)$ between the PR and P1 time points, which were increased at the P7 time point. (D) Pathway analysis between the PR and P1 time points $(-\log (\mathrm{P})>1)$. Unclear pathways are not presented. (E) Pathway analysis between the $\mathrm{P} 1$ and $\mathrm{P} 7$ time points $(-\log (\mathrm{P})>1)$. CGs, CG sites; FC, fold-change; PR, prior to thermal therapy; P1, 1-3 days post-ablation; P7, 5-7 days post-ablation.

pathways altered between the PR and $\mathrm{P} 7$ time points $(-\log (\mathrm{P})>1$, at least three genes in one pathway). In total, 7 pathways were activated, including innate immunity ('dendritic cell maturation' and 'production of nitric oxide and reactive oxygen species in macrophages'). In addition, T cell-related pathways were also observed, such as 'CXCR4 signaling', the 'Th1 pathway' and the 'Th2 pathway'. The 'osteoarthritis pathway' was suppressed, and the change trends for 21 other pathways were unclear (Fig. 3A).

Excluding the T helper cell 1 (Th1), T helper cell 2 (Th2) and neuroinflammation pathways, the present study focused on the remaining four pathways ('p70S6K signaling', 'CXCR4 signaling', 'dendritic cell maturation' and 'production of nitric oxide and reactive oxygen species in macrophages'), to further analyze the increased or decreased expression of the pathway associated CGs (adjusted $\mathrm{P}<0.05$; $|\log (\mathrm{FC})|>0.5$ ), and the upregulated or downregulated expression of the annotated genes (Fig. 3B-E). The majority of CGs had consistent change trends that there was an increase at the P1 time point, there was also an increase at the $\mathrm{P} 7$ time points; there was a decrease at the $\mathrm{P} 1$ time point, there was also a decrease at the P7 time points (Fig. 3B-E).

\section{Discussion}

An effective tumor-specific immune response and long-lasting anti-tumor immunity is necessary for the development of an effective form of immunotherapy (9). The present study focused on the global genomic DNA methylation profiles of PBMCs to describe the overall immune change trends following ablation treatment for patients with $\mathrm{HBV}$-associated
HCC. DNA methylation profiles of pathways associated with the immune system were different at the P1 and P7 time points following ablation, and affected the systemic stress response at the P1 time point in particular. The activation of 'Th1 pathway', 'Th2 pathway' and 'CXCR4 signaling', as well as macrophage and dendritic cell-related pathways indicated that effective immune responses were observable at the P7 time point. However, DNA methylation in these pathways were already altered at the P1 time point, suggesting that changes in DNA methylation occurred early after ablation. Hence, the timing of combined immunotherapy and ablation procedures should be chosen with caution.

As a main site of immune tolerance, the liver can affect pathogen-specific immune responses (27). In addition, the liver can release $\mathrm{HCC}$-associated circular tumor DNA to the peripheral blood $(28,29)$. Abnormal liver lesions can also be detected by clinical laboratory tests, such as alanine transaminase and aspartate aminotransferase. Our previous study revealed that alterations of DNA methylation in the immune system were closely associated with the development of HCC (19). Other previous studies suggested that ablation could induce tumor antigen-associated $\mathrm{T}$ cell responses (10), as well as systemic immune responses (12). As peripheral immune cells can reflect the HCC-associated immune response to a certain extent, PBMCs were used for analysis in the present study. Following different methylation CGs analysis and the estimation of gene expression, the methylation levels of those CGs and immune-associated pathways were used to observe the methylation and immune 


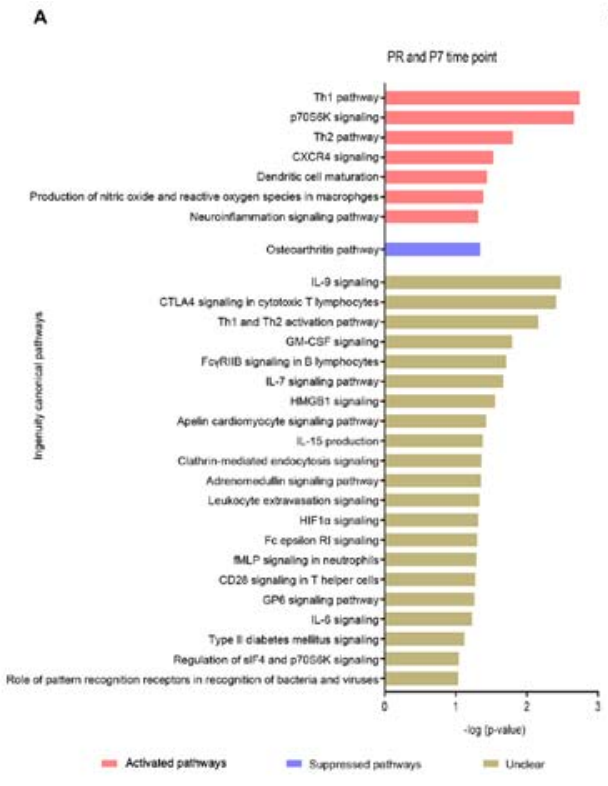

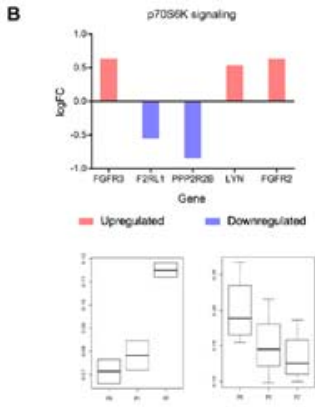

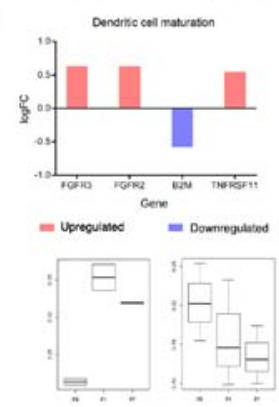

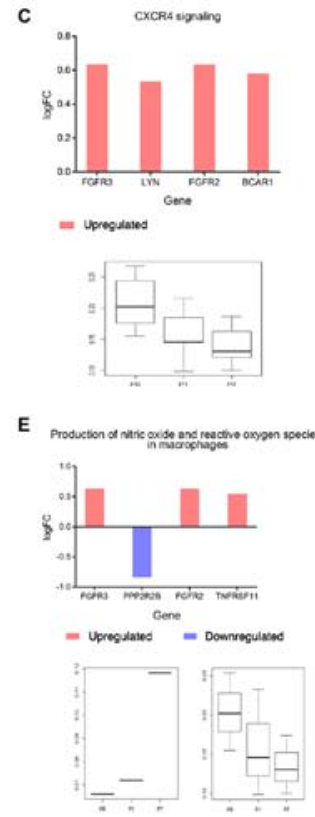

Figure 3. Pathway analysis between the PR and P7 time points. (A) Pathway analysis between the PR and P7 time points (-log(P) $>1)$. (B) Change trend of 'p30S6K signaling'-associated genes between PR and P7 time points (upper). Red indicates activation, blue indicates suppression. Boxplot (bottom) indicates the mean methylation levels of increased (left) and decreased (right) CGs in this pathway, whose levels of methylation were significant between PR and P7 time points (adjusted $\mathrm{P}<0.05$; $|\log (\mathrm{FC})|>0.5$ ). (C) Change trend of 'CXCR4 signaling'-associated genes between PR and P7 time points (upper), red indicates activation. Boxplot (bottom) shows the mean methylated levels of those decreased CGs in this pathway, whose levels of methylation were significant between PR and P7 time points (adjusted P $<0.05$; $|\log (\mathrm{FC})|>0.5$ ). (D) Change trend of 'dendritic cell maturation' pathway-associated genes between PR and P7 time points (upper), red indicates activation, blue indicates suppression. Boxplot (bottom) indicates the mean methylation levels of increased (left) and decreased (right) CGs in this pathway, whose levels of methylation were significant between PR and P7 time points (adjusted P<0.05; Ilog(FC)l>0.5), respectively. (E) Change trend of 'production of nitric oxide and reactive oxygen species in macrophages'-associated genes between PR and P7 time points (upper). Red indicates activation, blue indicates suppression. Boxplot (bottom) indicates the mean methylation levels of increased (left) and decreased (right) CGs in this pathway, whose levels of methylation were significant between PR and P7 time points (adjusted P<0.05; |log(FC)l>0.5). FC, fold-change; CGs, CG sites; PR, prior to thermal therapy; P1, 1-3 days post-ablation; P7, 5-7 days post-ablation.

response change trend following ablation. The increased sites between the PR and P1 time points gradually decreased at the P7 time point, and the decreased sites between the PR and $\mathrm{P} 1$ time points gradually increased at the P7 time point. Combined with the pathway analysis, which demonstrated that the activated pathways between the PR and P1 time points were suppressed at the P1 and P7 time points, and the stress response-associated 'adrenomedullin signaling pathway' and 'NRF2-mediated oxidative stress response' were also suppressed at the P1 and P7 time points. Therefore, the present study suggested that the suppressed effect of those pathways was caused by a systemic stress response.

Between the PR and P7 time points, 7 signaling pathways were activated following ablation. Among them, the neuroinflammation and osteoarthritis pathways $(30,31)$, Th1, Th2 and p70 ribosomal S6 kinase (p70S6K) signaling were associated with the change of immune microenvironment. Previous studies about HCC indicated that Th1 cells mediated the immune clearance of tumor cells and prevented tumorigenesis (32), and the cytokines that they produced are strongly associated with good clinical outcome (33), whereas Th2 cells were associated with tumor growth or metastasis (34). It is well known that Th1/Th2 balance is broken in the HCC micro-environment (35). Thus, the activation of Th1 and Th2 signaling pathways and the significant activation of Th1 pathway in the present study suggested the possibility that ablation may reverse the Th1/Th2 immune imbalance of HCC subjects. p70S6K is a serine/threonine protein kinase, and the activation of the $\mathrm{PI} 3 \mathrm{~K} / \mathrm{mTOR} / \mathrm{p} 70 \mathrm{~S} 6 \mathrm{~K}$ pathway may serve to integrate the extracellular signals to regulate the appropriate differentiation program of naïve $\mathrm{CD}^{+} \mathrm{T}$ cells and the generation of effector T cells (36). Robust Th1 cell and cytotoxic immune responses are associated with prolonged survival in patients with HCC (33). Based on the aforementioned data, it can be speculated that the activation of Th1 and p70S6K pathways after ablation were associated with immune activation.

C-X-C motif chemokine receptor type 4 (CXCR4) is an $\alpha$-chemokine receptor that binds stromal-derived factor 1 and can activate multiple biological processes (37). Following $\mathrm{T}$ cell receptor crosslinking, CXCR4 is recruited and accumulates at the immunological synapse, resulting in a stronger $\mathrm{T}$ cell/antigen-presenting cell (APC) interaction, high levels of T cell proliferation and interferon- $\gamma$ production (38). Meanwhile, the activation of classically activated macrophages can produce pro-inflammatory cytokines and reactive oxygen/nitrogen species, which are crucial for host defense and tumor cell killing, and suppress HCC cell growth and induce liver tumor regression (39). Phenotypically, they also express high levels of major histocompatibility complex class II, CD68, CD80 and CD86 costimulatory molecules (40). In addition, tumor-specific $\mathrm{T}$ cells in pre-malignant liver lesions encountering tumor antigens on non-professional and/or non-activated APCs in a non-inflammatory context can lead to peripheral self-tolerance (14). Therefore, the activation of CXCR4, dendritic cell maturation and macrophage signaling can promote the interaction between 
T cell and APC, increase host defense function, and are necessary for effective anti-tumor immune responses.

A correlation analysis among the PR, P1 and P7 time points demonstrated that the M3 and M4 subjects had a different change in trend compared with others (data not shown). Considering the consistency of the inclusion and exclusion criteria, the potential confounding factors (such as smoking, drinking, sex and age) can be ignored by paired analysis, so the ablation and tumor-associated factor may be the main factors accounting for this difference. Previous studies indicated that incomplete ablation could activate signaling pathways that may favor pro-tumor development or metastasis (41-43). In the present study, two subjects (M3 and M4) received incomplete ablation at the sample collection time point. According to the aforementioned analysis, the present study inferred that these two subjects with incomplete ablation may have a different immune response trend compared with the complete ablation subjects, however, this requires further validation.

According to the standard deviation among three time points (0.016) and the desired power $(0.8)$, the appropriate sample sizes for microarray experiments were calculated, which suggested that a small sample size $(n=3)$ was required to detect a $\delta$ of 0.1 at the microarray data set. Thus, the paired sample size of five is large enough to explain those results. However, there were several limitations to the present study. First, since there were only five subjects in the present study, the immune response could not be compared between incomplete and complete ablation subjects. Secondly, gene expression is affected not only by DNA methylation, but also by histone deacetylation (44). Thus, the effects of deacetylation should also be investigated. Thirdly, a previous study indicated that a single high dose of radiation could affect the immune response (45) In the present study, samples were collected before and after the first ablation treatment to understand how the immune response is affected by repeated ablation treatments requires further investigation.

Overall, the results of the present study demonstrated that DNA methylation could be altered soon after ablation, while systemic immune responses were induced at the P7 time point. Furthermore, the continuously activated signaling pathways may not end at the P7 time points. Hence, it is necessary to investigate the duration of immune activation, and the time point of immunotherapy, particularly for the immune checkpoint inhibitor for ablation subjects in a future study.

\section{Acknowledgements}

Not applicable.

\section{Funding}

The present study was supported by The Beijing Natural Science Foundation (grant no. 7191004), The Beijing Municipal Science \& Technology Commission (grant no. Z171100001017078), The Beijing Municipal Administration of Hospitals (grant nos. DFL20181701 and ZYLX201711), The National S \& T Major Project for Infectious Diseases Control (grant no. 2017ZX10201101001008) and The Biomarkers of Infection-Related Diseases Beijing Key Laboratory (grant no. BZ0373). The funders had no role in study design, data collection and analysis, decision to publish, or preparation of the manuscript.

\section{Availability of data and materials}

The datasets used and/or analyzed during the present study are available from the corresponding author upon reasonable request.

\section{Authors' contributions}

YNZ performed the experiments, data analysis, writing of the original draft and reviewing the manuscript. KL and JS performed the experiments and data analysis. JS also wrote and reviewed the manuscript for important intellectual content. $\mathrm{NH}$ and $\mathrm{PZ}$ performed the quality control of the data and algorithms, and the clinical data evaluation. $\mathrm{CZ}, \mathrm{XY}, \mathrm{CH}, \mathrm{JL}, \mathrm{HZ}$ and QW collected the clinical samples and performed the clinical data evaluation. YHZ and YZ conceptualized and designed the present study, acquired the funding, performed the project administration, drafted the original manuscript and reviewed the manuscript for important intellectual content. All authors have read and approved the final version of the manuscript.

\section{Ethics approval and consent to participate}

The present study was approved by The Ethics Committee of Beijing You'an Hospital, Capital Medical University. Written informed consent was obtained from all subjects. The present study was performed according to the guidelines of the Helsinki Declaration [ethical approval no. JingyouKelunzi (2017) no. 26].

\section{Patient consent for publication}

Not applicable.

\section{Competing interests}

The authors declare that they have no competing interests.

\section{References}

1. Bray F, Ferlay J, Soerjomataram I, Siegel RL, Torre LA and Jemal A: Global cancer statistics 2018: GLOBOCAN estimates of incidence and mortality worldwide for 36 cancers in 185 countries. CA Cancer J Clin 68: 394-424, 2018.

2. Feng RM, Zong YN, Cao SM and Xu RH: Current cancer situation in China: Good or bad news from the 2018 Global cancer statistics? Cancer Commun (Lond) 39: 22, 2019.

3. Liu J, Liang W, Jing W and Liu M: Countdown to 2030: Eliminating hepatitis B disease. China. Bull World Health Organ 97: 230-238, 2019.

4. Chen MS, Li JQ, Zheng Y, Guo RP, Liang HH, Zhang YQ, Lin XJ and Lau WY: A prospective randomized trial comparing percutaneous local ablative therapy and partial hepatectomy for small hepatocellular carcinoma. Ann Surg 243: 321-328, 2006.

5. Jiang Y, Zhou S, Shen G, Jiang H and Zhang J: Microwave ablation combined with transcatheter arterial chemoembolization is effective for treating unresectable hepatoblastoma in infants and children. Medicine (Baltimore) 97: e12607, 2018.

6. Tang C, Shen J, Feng W, Bao Y, Dong X, Dai Y, Zheng Y and Zhang J: Combination therapy of radiofrequency ablation and transarterial chemoembolization for unresectable hepatocellular carcinoma: A retrospective study. Medicine (Baltimore) 95: e3754, 2016. 
7. Wang WD, Zhang LH, Ni JY, Jiang XY, Chen D, Chen YT, Sun HL, Luo JH and Xu LF: Radiofrequency ablation combined with transcatheter arterial chemoembolization therapy versus surgical resection for hepatocellular carcinoma within the milan criteria: A meta-analysis. Korean J Radiol 19: 613-622, 2018

8. Hocquelet A, Balageas P, Laurent C, Blanc JF, Frulio N, Salut C, Cassinotto C, Saric J, Possenti L, Bernard PH, et al Radiofrequency ablation versus surgical resection for hepatocellular carcinoma within the milan criteria: A study of 281 Western patients. Int J Hyperthermia 31: 749-757, 2015.

9. Greten TF, Manns MP and Korangy F: Immunotherapy of hepatocellular carcinoma. J Hepatol 45: 868-878, 2006.

10. Mizukoshi E, Yamashita T, Arai K, Sunagozaka H, Ueda T, Arihara F, Kagaya T, Yamashita T, Fushimi K and Kaneko S: Enhancement of tumor-associated antigen-specific $\mathrm{T}$ cell responses by radiofrequency ablation of hepatocellular carcinoma. Hepatology 57: 1448-1457, 2013.

11. Zerbini A, Pilli M, Penna A, Pelosi G, Schianchi C, Molinari A, Schivazappa S, Zibera C, Fagnoni FF, Ferrari C and Missale G: Radiofrequency thermal ablation of hepatocellular carcinoma liver nodules can activate and enhance tumor-specific T-cell responses. Cancer Res 66: 1139-1146, 2006.

12. Waitz R and Solomon SB: Can local radiofrequency ablation of tumors generate systemic immunity against metastatic disease. Radiology 251: 1-2, 2009.

13. Youngblood B, Hale JS, Kissick HT, Ahn E, Xu X, Wieland A, Araki K, West EE, Ghoneim HE, Fan Y, et al: Effector CD8 T cells dedifferentiate into long-lived memory cells. Nature 552: 404-409, 2017.

14. Schietinger A, Philip M, Krisnawan VE, Chiu EY, Delrow JJ, Basom RS, Lauer P, Brockstedt DG, Knoblaugh SE, Hämmerling GJ, et al: Tumor-specific T cell dysfunction is a dynamic antigen-driven differentiation program initiated early during tumorigenesis. Immunity 45: 389-401, 2016.

15. Gao W, Kondo Y, Shen L, Shimizu Y, Sano T, Yamao K, Natsume A, Goto Y, Ito M, Murakami H, et al: Variable DNA methylation patterns associated with progression of disease in hepatocellular carcinomas. Carcinogenesis 29: 1901-1910, 2008.

16. Ye C, Tao R, Cao Q, Zhu D, Wang Y, Wang J, Lu J, Chen E and Li L: Whole-genome DNA methylation and hydroxymethylation profiling for HBV-related hepatocellular carcinoma. Int J Oncol 49: 589-602, 2016.

17. Ahn E, Youngblood B, Lee J, Lee J, Sarkar S and Ahmed R: Demethylation of the PD-1 promoter is imprinted during the effector phase of Cd8 T cell exhaustion. J Virol 90: 8934-8946, 2016.

18. Ghoneim HE, Fan Y, Moustaki A, Abdelsamed HA, Dash P, Dogra P, Carter R, Awad W, Neale G, Thomas PG and Youngblood B: De novo epigenetic programs inhibit PD-1 blockade-mediated t cell rejuvenation. Cell 170: 142-157 e19, 2017.

19. Zhang Y, Petropoulos S, Liu J, Cheishvili D, Zhou R, Dymov S, Li K, Li N and Szyf M: The signature of liver cancer in immune cells DNA methylation. Clin Epigenetics 10: 8, 2018.

20. European Association for the Study of the Liver. Electronic address; easloffice@easloffice.eu: Corrigendum to 'EASL clinical practice guidelines: Management of hepatocellular carcinoma' [J Hepatol 69 (2018) 182-236]. J Hepatol 70: 817, 2019.

21. Forner A, Reig M and Bruix J: Hepatocellular carcinoma. Lancet 391: 1301-1314, 2018

22. Morris TJ, Butcher LM, Feber A, Teschendorff AE, Chakravarthy AR, Wojdacz TK and Beck S: ChAMP: $450 \mathrm{k}$ Chip analysis methylation pipeline. Bioinformatics 30: 428-430, 2014.

23. Ritchie ME, Phipson B, Wu D, Hu Y, Law CW, Shi W and Smyth GK: Limma powers differential expression analyses for RNA-sequencing and microarray studies. Nucleic Acids Res 43: e47, 2015 .

24. Aryee MJ, Jaffe AE, Corrada-Bravo H, Ladd-Acosta C, Feinberg AP, Hansen KD and Irizarry RA: Minfi: A flexible and comprehensive bioconductor package for the analysis of infinium DNA methylation microarrays. Bioinformatics 30: 1363-1369, 2014.

25. Maksimovic J, Phipson B and Oshlack A: A cross-package bioconductor workflow for analysing methylation array data. Version 3. F1000Res 5: 1281, 2016.
26. Leek JT, Johnson WE, Parker HS, Jaffe AE and Storey JD: The sva package for removing batch effects and other unwanted variation in high-throughput experiments. Bioinformatics 28: 882-883, 2012

27. Jenne $\mathrm{CN}$ and Kubes P: Immune surveillance by the liver. Nat Immunol 14: 996-1006, 2013

28. Sun K, Jiang P, Chan KC, Wong J, Cheng YK, Liang RH, Chan WK, Ma ES, Chan SL, Cheng SH, et al: Plasma DNA tissue mapping by genome-wide methylation sequencing for noninvasive prenatal, cancer, and transplantation assessments. Proc Natl Acad Sci USA 112: E5503-E5512, 2015.

29. Xu RH, Wei W, Krawczyk M, Wang W, Luo H, Flagg K, Yi S, Shi W, Quan Q, Li K, et al: Circulating tumour DNA methylation markers for diagnosis and prognosis of hepatocellular carcinoma. Nat Mater 16: 1155-1161, 2017.

30. Molfino A, Gioia G, Rossi Fanelli F and Laviano A: Contribution of neuroinflammation to the pathogenesis of cancer cachexia. Mediators Inflamm 2015: 801685, 2015.

31. Haywood L, McWilliams DF, Pearson CI, Gill SE, Ganesan A, Wilson D and Walsh DA: Inflammation and angiogenesis in osteoarthritis. Arthritis Rheum 48: 2173-2177, 2003.

32. Mossanen JC and Tacke F: Role of lymphocytes in liver cancer. Oncoimmunology 2: e26468, 2013.

33. Fridman WH, Pages F, Sautes-Fridman C and Galon J: The immune contexture in human tumours: Impact on clinical outcome. Nat Rev Cancer 12: 298-306, 2012.

34. Lee HL, Jang JW, Lee SW, Yoo SH, Kwon JH, Nam SW, Bae SH, Choi JY, Han NI and Yoon SK: Inflammatory cytokines and change of Th1/Th2 balance as prognostic indicators for hepatocellular carcinoma in patients treated with transarterial chemoembolization. Sci Rep 9: 3260, 2019.

35. Cao X: Immunology in China: The past persent and future. Nat Immunol 9: 339-342, 2008

36. O'Brien TF and Zhong XP: The role and regulation of mTOR in T-lymphocyte function. Arch Immunol Ther Exp (Warsz) 60: 173-181, 2012.

37. Chatterjee S, Behnam Azad B and Nimmagadda S: The intricate role of CXCR4 in cancer. Adv Cancer Res 124: 31-82, 2014.

38. Eckert F, Schilbach K, Klumpp L, Bardoscia L, Sezgin EC, Schwab M, Zips D and Huber SM: Potential role of CXCR4 targeting in the context of radiotherapy and immunotherapy of cancer. Front Immunol 9: 3018, 2018.

39. Guerra AD, Yeung OWH, Qi X, Kao WJ and Man K: The anti-tumor effects of M1 macrophage-loaded poly (ethylene glycol) and gelatin-based hydrogels on hepatocellular carcinoma. Theranostics 7: 3732-3744, 2017.

40. Aras S and Zaidi MR: TAMeless traitors: Macrophages in cancer progression and metastasis. Br J Cancer 117: 1583-1591, 2017.

41. Dong S, Kong J, Kong F, Kong J, Gao J, Ke S, Wang S, Ding X, Sun W and Zheng L: Insufficient radiofrequency ablation promotes epithelial-mesenchymal transition of hepatocellular carcinoma cells through Akt and ERK signaling pathways. J Transl Med 11: 273, 2013.

42. Yuan CW, Wang ZC, Liu K and Liu DJ: Incomplete radiofrequency ablation promotes the development of $\mathrm{CD} 133^{+}$cancer stem cells in hepatocellular carcinoma cell line HepG2 via inducing SOX9 expression. Hepatobiliary Pancreat Dis Int 17: 416-422, 2018

43. Zhang N, Ma D, Wang L, Zhu X, Pan Q, Zhao Y, Zhu W, Zhou J, Wang L, Chai Z, et al: Insufficient radiofrequency ablation treated hepatocellular carcinoma cells promote metastasis by up-regulation ITGB3. J Cancer 8: 3742-3754, 2017.

44. Attwood JT, Yung RL and Richardson BC: DNA methylation and the regulation of gene transcription. Cell Mol Life Sci 59: 241-257, 2002 .

45. Zeng H, Zhang W, Gong Y and Xie C: Radiotherapy activates autophagy to increase $\mathrm{CD} 8^{+} \mathrm{T}$ cell infiltration by modulating major histocompatibility complex class-I expression in non-small cell lung cancer. J Int Med Res 47: 3818-3830, 2019.

This work is licensed under a Creative Commons Attribution-NonCommercial-NoDerivatives 4.0 International (CC BY-NC-ND 4.0) License. 